\title{
The pattern of involvement of the gastric mucosa in lymphocytic gastritis is predictive of the presence of duodenal pathology
}

\author{
M Hayat, D S Arora, J I Wyatt, S O’Mahony, M F Dixon
}

\begin{abstract}
Aim-To determine whether the pattern of involvement of the gastric mucosa in lymphocytic gastritis is predictive of the presence or absence of duodenal pathology.

Methods-50 cases (M:F, 26:24; median age 57 years) diagnosed as lymphocytic gastritis between 1986 and 1998 with concurrent duodenal (D2) biopsies were identified from a computer search of the pathology records and validated by counting gastric intraepithelial lymphocytes. Gastric and duodenal intraepithelial lymphocyte counts were performed on haematoxylin and eosin (H\&E) and anti-CD3 stained sections. D2 biopsies were assessed for villous atrophy and chronic inflammatory cell infiltration by subjective grading, and gastritis was classified and graded according to the updated Sydney system. A case was designated corpus predominant when the corpus chronic inflammation grade exceeded that of the antrum. If it was less, then the case was antrum predominant, and if they were equal it was diffuse (pan-) gastritis. The ratio between the corpus and antral intraepithelial lymphocyte count in individual patients was calculated.
\end{abstract}

Results-Of 50 cases of lymphocytic gastritis, 21 were classified as corpus predominant. With one exception (a case of mild villous atrophy), all were accompanied by normal duodenal morphology. Cases with a corpus predominant gastritis had median duodenal intraepithelial lymphocyte counts of 19 (H\&E) and 14.1 (CD3), whereas 29 subjects with an antrum predominant or diffuse gastritis had median counts of 39.9 (H\&E) and 37.9 (CD3). Fifteen of these 29 cases (52\%) showed villous atrophy; all were graded as moderate or severe. Patients with any degree of villous atrophy had a mean corpus/antrum intraepithelial lymphocyte ratio $(\mathrm{H} \& \mathrm{E})$ of 0.59 (representing antral predominance), while those with normal duodenal morphology had a ratio of 2.39 $(\mathrm{p}<0.0001)$.

Conclusions-The pattern of involvement of gastric mucosa in lymphocytic gastritis is closely related to the associated duodenal pathology. Those with the corpus predominant form are unlikely to have duodenal pathology, while those with an antral predominant or diffuse form should have distal duodenal biopsies taken to exclude villous atrophy.

(F Clin Pathol 1999;52:815-819)

Keywords: lymphocytic gastritis; intraepithelial lymphocytosis; villous atrophy; coeliac disease

Lymphocytic gastritis is characterised by an increase in intraepithelial lymphocytes in surface and foveolar epithelium, together with a variable increase in chronic inflammatory cells in the lamina propria of the gastric mucosa. ${ }^{12}$ Although the degree of intraepithelial lymphocytosis shows patchy variation, the disorder usually affects the whole stomach. It is found in $0.83-2.5 \%$ of unselected patients undergoing upper gastrointestinal endoscopy ${ }^{3}$ and in $4.5 \%$ of those with histological chronic gastritis. ${ }^{4}$ This type of gastritis may be associated with a normal looking gastric mucosa at endoscopy. However, the classical endoscopic appearances comprise prominent rugal folds bearing small nodular elevations surmounted by small grey-white erosions with hyperaemic margins, maximal in the body and fundus. ${ }^{14}$ This endoscopic picture is generally termed "varioliform" gastritis.

The aetiology of lymphocytic gastritis is not known. It has been suggested that it represents an idiosyncratic immune response to some local antigen, such as $H$ pylori. ${ }^{45}$ However, an increased density of intraepithelial lymphocytes can also be found in the gastric mucosa of patients with coeliac disease and in a proportion their numbers is sufficient to diagnose lymphocytic gastritis. ${ }^{67}$ This coeliac disease associated form of lymphocytic gastritis is usually not associated with the endoscopic picture of varioliform gastritis. An observation made by one of us (JIW) suggested that the pattern of involvement of the gastric mucosa in lymphocytic gastritis associated with coeliac disease may be different from the form not associated with coeliac disease (isolated lymphocytic gastritis).

The aim of the present study was to determine whether the pattern of involvement of the gastric mucosa in lymphocytic gastritis is predictive of the presence of duodenal pathology.

\section{Methods}

HISTOLOGY

Fifty nine cases diagnosed as lymphocytic gastritis between 1986 and 1998 with duodenal (second part, D2) biopsies were identified from a computer search of the pathology records. 
Specimens were fixed in 10\% buffered formalin, embedded in paraffin wax, and sections were stained with haematoxylin and eosin $(\mathrm{H} \& \mathrm{E})$ and a modified Giemsa stain for the detection of $H$ pylori. The original diagnosis was validated by performing counts of gastric intraepithelial lymphocytes on both the antrum and corpus biopsies. H\&E stained sections were examined at $\times 400$ magnification and the numbers of intraepithelial lymphocytes and epithelial cell nuclei in an uninterrupted length of surface and foveolar epithelium ( $>500$ cells) were counted (by $\mathrm{MH}$ ). Based on previous studies, ${ }^{12}$ a count of 25 intraepithelial lymphocytes per 100 epithelial cells in the areas of maximum lymphocytic infiltration was used as the lower limit for inclusion of a case into this study. In nine of 59 cases intraepithelial lymphocytes were insufficient for the diagnosis of lymphocytic gastritis, so they were excluded from the study. Of the 50 cases included, 26 were male and 24 female, with a median age of 57 years. In these 50 cases, intraepithelial lymphocyte counts were then performed on the duodenal biopsies. In a separate exercise, the severity of gastritis in the antral and corpus biopsies was graded according the updated Sydney system ${ }^{8}$ and D2 biopsies were assessed for villous atrophy and chronic inflammatory cell infiltration by subjective grading by an experienced pathologist (MFD). Gastric biopsies were graded before looking at the duodenal biopsies to improve "blinding." The grade of chronic inflammation in the lamina propria of the gastric mucosa ( $0-3$ equivalent to absent, mild, moderate, or marked) in the paired antral and corpus biopsies was used to determine the pattern of gastritis.

\section{IMMUNOHISTOLOGY}

Further sections of the duodenal and gastric biopsy specimens were immunostained using a three step immunoperoxidase technique with anti-CD3 (pan T cell) monoclonal antibodies (Dako). CD3 positive intraepithelial lymphocyte counts were performed as before but by a second observer (DSA).

TOPOGRAPHY OF GASTRITIS

The topography of gastritis was described in two ways, one being the conventional antrum versus corpus predominant, based on the severity of chronic inflammatory cell infiltration; and the other being the relation of intraepithelial lymphocytes counts in the two compartments. To designate a case as corpus predominant, the corpus chronic inflammation grade must exceed that of antrum. If it is less,

Table 1 Intraepithelial lymphocyte counts (median and range) at three mucosal sites (HE E and anti-CD3 stained sections) from patients with lymphocytic gastritis $(L G)$ and normal duodenal morphology (isolated $L G$ ), and lymphocytic gastritis with villous atrophy (VA)

\begin{tabular}{llll}
\hline Site & Stain & Isolated $L G(n=34)$ & $L G+V A(n=16)$ \\
\hline Antrum & H\&E & $23.0(7.1$ to 61.0$)$ & $38.0(15.0$ to 71.2$)$ \\
\multirow{2}{*}{ Corpus } & CD3 & $17.4(3.6$ to 58.3$)$ & $28.5(14.8$ to 57.6$)$ \\
\multirow{2}{*}{ Duodenum } & H\&E & $45.0(6.6$ to 96.0$)$ & $22.8(9.0$ to 33.2$)$ \\
& CD3 & $41.2(8.2$ to 107.2$)$ & $22.2(3.3$ to 34.3$)$ \\
& H\&E & $19.0(4.9$ to 45.0$)$ & $39.9(16.0$ to 73.5$)$ \\
& CD3 & $14.1(4.9$ to 36.2$)$ & $37.9(19.7$ to 99.1$)$ \\
\hline
\end{tabular}

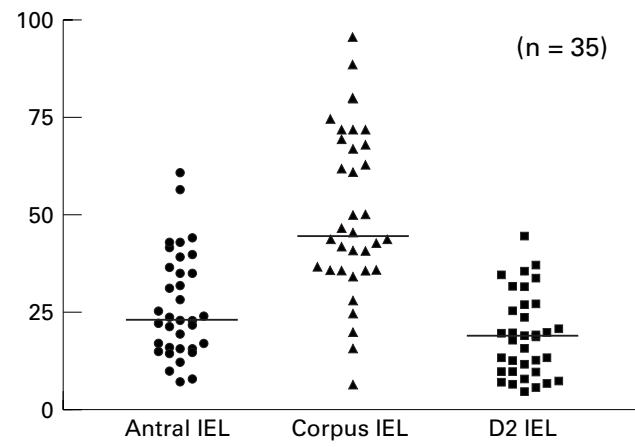

Figure 1 Intraepithelial lymphocyte (IEL) counts in antral, corporal, and duodenal biopsies from patients with lymphocytic gastritis and normal duodenal morphology.

then the case is antrum predominant and if they are equal it is diffuse (pan-) gastritis. The ratio between corporal and antral intraepithelial lymphocyte counts (H\&E) was calculated.

DEGREE OF VILLOUS ATROPHY

The degree of villous atrophy was assessed by subjective grading on a $0-3$ scale where $0=$ absent (normal) and 1 to 3 represent mild, moderate, and marked villous atrophy. Duodenal biopsies were also graded 0 to 3 for chronic inflammatory cell infiltration of the lamina propria.

\section{STATISTICS}

Results are expressed as medians and means (with ranges). Variables were compared using the $\chi^{2}$ (two tailed) and the Mann-Whitney U test (two tailed). Correlation between H\&E and CD3 counts was assessed using Spearman's test (two tailed). The criterion for statistical significance was defined as a level of $\mathrm{p}<0.05$.

\section{Results}

In 34 patients with lymphocytic gastritis the duodenal villous morphology was considered to be normal, while 16 patients had some degree of villous atrophy. $H$ pylori was detected on histology in 14 of the 50 cases. Of these, nine had normal duodenal histology while five had villous atrophy.

PATIENTS WITH LYMPHOCYTIC GASTRITIS AND NORMAL DUODENAL BIOPSIES

In this group of patients the median grade of chronic inflammation in the antrum was 1 (1 to 2 ), in the corpus 2 ( 1 to 3 ), and 0 in the duodenum. There was no difference in the chronic inflammatory grades in $H$ pylori positive versus negative patients.

The median intraepithelial lymphocyte counts on H\&E stained sections are shown in table 1 and fig 1 . The mean corpus/antrum intraepithelial lymphocyte ratio (H\&E) was 2.39 (0.30 to 9.00$)$.

\section{PATIENTS WITH LYMPHOCYTIC GASTRITIS AND} VILLOUS ATROPHY

Sixteen patients had duodenal villous atrophy with a median grade of 2.5 ( 1 to 3 ), and a median grade of $2(0-3)$ for chronic inflamma- 


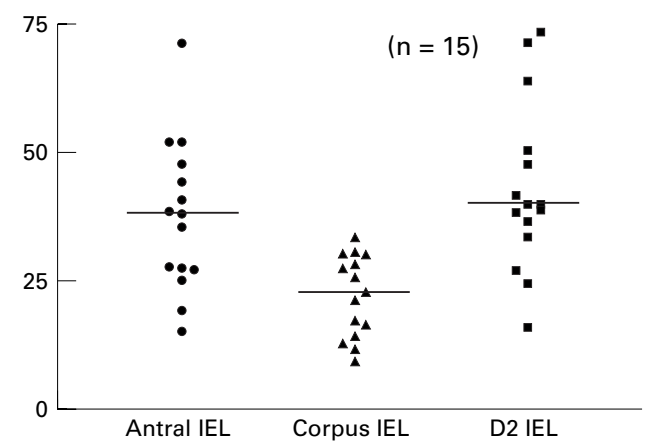

Figure 2 Intraepithelial lymphocyte (IEL) counts in antral, corpus, and duodenal biopsies from patients with lymphocytic gastritis and villous atrophy.

tory cell infiltration in the duodenal mucosa. Gastric chronic inflammatory cell infiltration had a median grade of 2 ( 1 to 2 ) in the antrum and 1 ( 0 to 2$)$ in the corpus. There was a mild increase in chronic inflammatory cell infiltration in the corpus mucosa (median 2 (1 to 2 )) in the $H$ pylori positive compared with $H$ pylori negative patients. However, there were only five positive patients with atrophy so one cannot draw any conclusions from this finding. The median intraepithelial lymphocyte counts are shown in table 1 and fig 2 . In general intraepithelial lymphocytes counts on H\&E exceeded those given by CD3 staining; the mean difference between the counts was $3.66 \%$. There was a significant correlation $(\mathrm{p}=0.01$, Spearman's two tailed coefficient, 0.249) between the H\&E and CD3 intraepithelial lymphocyte counts. The mean corpus/antrum intraepithelial lymphocytes ratio in the villous atrophy group was 0.59 (0.20 to 1.20$)$.

PATTERN OF GASTRITIS

Of the 50 cases of lymphocytic gastritis, 21 were classified as corpus predominant (fig 3 ). With only one exception (a case with mild villous atrophy), all were accompanied by normal duodenal morphology (table 2). Of those with an antral predominant or diffuse gastritis pattern, 52\% (15 of 29) had some degree of
Table 2 Distribution of 50 cases of lymphocytic gastritis according to pattern of gastritis and villous morphology

\begin{tabular}{llll}
\hline \multirow{2}{*}{$\begin{array}{l}\text { Lymphocytic gastritis } \\
\text { present }\end{array}$} & \multicolumn{2}{l}{ Villous atrophy } & \\
\cline { 2 - 3 } & Present & Absent & Total \\
\hline Antral predominant or & & & \\
$\quad$ pangastritis & 15 & 14 & 29 \\
Corpus predominant & 1 & 20 & 21 \\
\hline$\chi^{2}=12.34$ (two tailed); $\mathrm{p}<0.001$. & &
\end{tabular}

villous atrophy (fig 4). Conversely, 15 of the 50 cases $(30 \%)$ showed moderate to severe villous atrophy; none of these cases had a corpus predominant gastritis.

When the pattern of lymphocytic gastritis was determined using the objective criterion of corpus to antrum (C/A) intraepithelial lymphocyte ratios, significant differences in the associated duodenal morphology emerged (table 3): of 31 patients with a C/A ratio $>1.0$ (representing greater lymphocytosis in the corpus), 29 (94\%) had a duodenal intraepithelial lymphocyte count of $<20$ and normal villous morphology; on the other hand, 13 of 18 patients $(72 \%)$ with a $\mathrm{C} / \mathrm{A}$ ratio $<1.0$ (greater lymphocytosis in the antrum) had a duodenal intraepithelial lymphocyte count of $>35$ and some degree of villous atrophy. However, there was no significant correlation between the intraepithelial lymphocyte counts and the chronic inflammation grade in antrum, corpus, and duodenum in the two groups (data not shown).

When the patients are divided according to whether or not there was a subjective diagnosis of villous atrophy (as above) the gastric C/A intraepithelial lymphocyte ratios in the two groups (mean 0.59 with villous atrophy $v 2.39$ without villous atrophy) are highly significantly different $(p<0.0001$, Mann-Whitney $U$ test (two tailed)).

\section{Discussion}

This study has confirmed our hypothesis that the pattern of involvement of the gastric mucosa in lymphocytic gastritis is predictive of

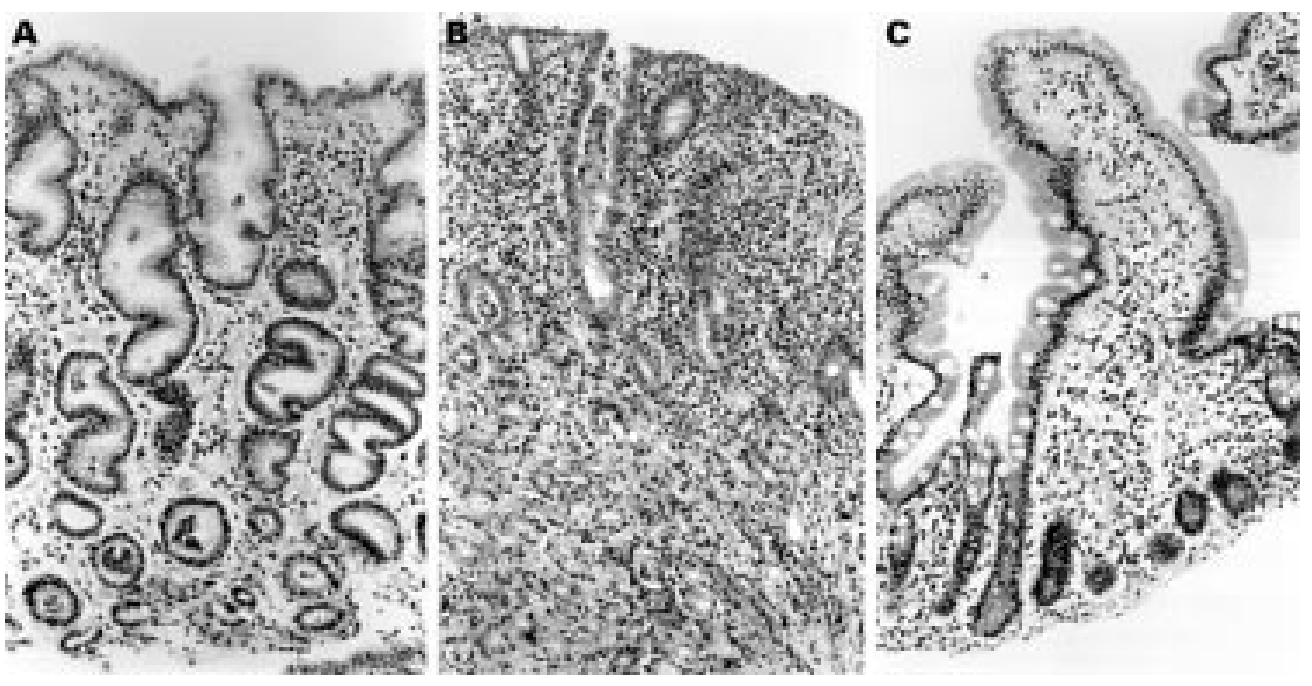

Figure 3 Antral (A), corporal (B), and duodenal (C) mucosa from a patient with corpus predominant lymphocytic gastritis and normal duodenal morphology. 
A
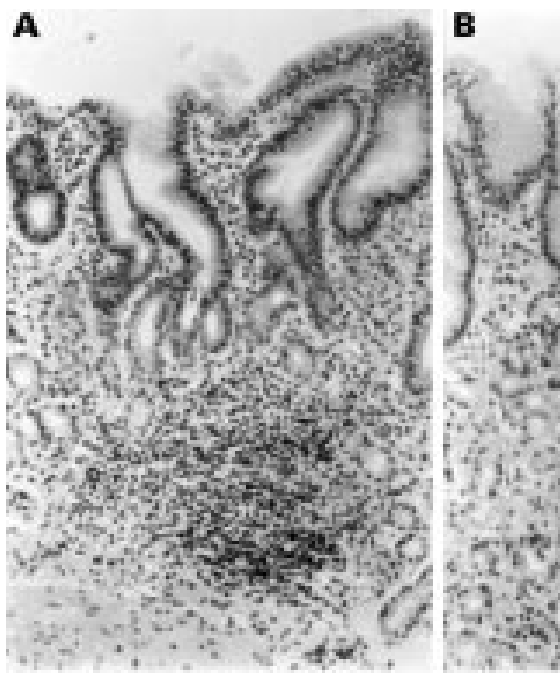

gastritis and villous atrophy.

Table 3 Distribution of cases of lymphocytic gastritis according to the ratio of intraepithelial lymphocyte (IEL) counts in the corpus and antrum and the duodenal IEL count

\begin{tabular}{llc}
\hline & \multicolumn{2}{l}{ Duodenal IEL count } \\
\cline { 2 - 3 } $\begin{array}{l}\text { Corpus/antrum } \\
\text { IEL ratio }\end{array}$ & $<20 / 100 E C^{\star}$ & $>35 / 100$ EC \\
\hline$>1.0$ & 29 & 2 \\
$<1.0$ & 5 & 13 \\
\hline
\end{tabular}

$\star$ In one patient with a median duodenal IEL count $<20$ there was insufficient surface epithelium in the corpus biopsies to perform and IEL count

$\chi^{2}=23.19$ (two tailed); $\mathrm{p}<0.001$.

EC, epithelial cells.

the presence of duodenal villous atrophy. Patients with classical, corpus predominant lymphocytic gastritis almost invariably have normal duodenal morphology, whereas those with an antrum predominant or diffuse pattern have a $50 \%$ chance of coexistent villous atrophy.

Interestingly, although the classification into an antrum or corpus predominant pattern was based on the degree of chronic inflammatory cell infiltration in the lamina propria, and this was borne out by intraepithelial lymphocyte ratios, there was no significant correlation between the two in individual patients. This disparity between the numbers of intraepithelial lymphocytes and the degree of lamina propria infiltration within patients has been mentioned in previous studies. ${ }^{14}$

Although the response to gluten withdrawal was not formally assessed in this study, the finding of villous atrophy in 15 of 50 cases $(30 \%)$ supports the view that coeliac disease has a close association with lymphocytic gastritis. Coeliac disease is characterised by lymphocytic infiltration not only in the small intestine, but also in the colon ${ }^{9}{ }^{10}$ and stomach. ${ }^{11}{ }^{12}$ Lymphocytic colitis occurs commonly in patients with "refractory" coeliac disease, ${ }^{13}$ and the rectal mucosa responds to gluten challenge with lymphocytic infiltration. ${ }^{14}$ It is possible that lymphocytic colitis is another manifestation of a mucosal immune response to a luminal antigen, which in this instance is maximally expressed in the large intestine. Coeliac disease, therefore, may be regarded as a diffuse lymphocytic gastroenteropathy which varies in expression from site to site.

The aetiology of lymphocytic gastritis in non-coeliac cases (isolated lymphocytic gastritis) is not known. It has been suggested that lymphocytic gastritis may represent an immune response to some local antigen, such as $H_{\text {pylori. }}{ }^{5}$ We have shown using multiple tests that many cases of isolated lymphocytic gastritis are associated with $H$ pylori infection, and that eradication of infection brings about significant reduction in gastric intraepithelial lymphocytic infiltration, corpus inflammation, and dyspeptic symptoms. ${ }^{15}$ Curiously, in most of these patients, $H$ pylori infection was diagnosed on the basis of serology alone, with histology and the carbon-13 urea breath test being negative. We have speculated that in such cases the organism may be present in small numbers and exist in a different (coccoid) form, and that isolated lymphocytic gastritis represents a specific immune response to the infection. This response may be genetically determined, as the majority of these patients with isolated lymphocytic gastritis possess the coeliac associated HLA DQB ${ }^{\star} 0201$ allele (unpublished observations).

Lymphocytic gastritis in non-coeliac patients may still be a subtle form of gluten sensitivity. A previous study from our unit showed abnormal intestinal permeability and high duodenal intraepithelial lymphocyte counts in non-coeliac patients with lymphocytic gastritis. ${ }^{16}$ It is now recognised that there is a spectrum of gluten induced intestinal changes, ranging from the classic "coeliac" lesion of subtotal villous atrophy to more subtle manifestations, such as a high duodenal intraepithelial lymphocyte count in the absence of villous atrophy. ${ }^{17}$ Thus coeliac disease represents a primary small intestinal disorder which is accompanied by intraepithelial lymphocytosis in the stomach, while lymphocytic gastritis is primarily a gastric disorder accompanied in many cases by intraepithelial lymphocytosis and functional disturbances in the small intestine. 
The function of intraepithelial lymphocytes in gastric mucosa remains elusive. In the small intestine they are considered to represent the first line of defence against invading pathogens. They are also potentially involved in the destruction of damaged epithelial cells through their ability to recognise altered antigens on epithelial cells, but their role in lymphocytic gastritis remains to be determined. Gastric intraepithelial lymphocytes in isolated lymphocytic gastritis seem to behave differently from those in coeliac associated cases. The former differs from its coeliac disease associated counterpart by having larger numbers of CD3+ intraepithelial lymphocytes, and more that are positive for $\mathrm{T}$ cell restricted intracellular antigen (TIA-1, a marker of resting T lymphocytes possessing cytolytic potential), and granzyme $\mathrm{B}(\mathrm{GrB}$, a marker of activated, cytotoxic $\mathrm{T}$ lymphocytes). ${ }^{18}$

CONCLUSIONS

In summary, we have shown that patients with a corpus predominant pattern of lymphocytic gastritis will rarely have associated villous atrophy. In this study only one of 21 patients (5\%) with this pattern of gastritis had villous atrophy and even then it was mild. On the other hand, a patient with an antrum predominant or pangastritic pattern of lymphocytic gastritis has a $50 \%$ chance of having villous atrophy. This observation has important clinical implications and all patients with the latter pattern should have distal duodenal biopsies taken to exclude the possibility of covert coeliac disease.

1 Haot J, Hamichi L, Wallez L, et al. Lymphocytic gastritis: a newly described entity: a retrospective endoscopic and histological study. Gut 1988;29:1258-64.
2 Haot J, Jouret A, Willette M, et al. Lymphocytic gastritisprospective study of its relationship with varioliform gastritis. Gut 1990;31:282-5.

3 Jaskiewicz K, Price SK, Zak J, et al. Lymphocytic gastritis in nonulcer dyspepsia. Dig Dis Sci 1991;36:1079-83.

4 Dixon MF, Wyatt JI, Burke DA, et al. Lymphocytic gastritis: relationship to Campylobacter pylori infection. $\mathcal{F}$ Pathol 1988;154:125-32.

5 Niemela S, Karttunen T, Kerola T, et al. Ten year follow up study of lymphocytic gastritis: further evidence on Helicobacter pylori as a cause of lymphocytic gastritis and corpus gastritis. F Clin Pathol 1995;48:1111-16.

6 Alsaigh N, Odze R, Goldman H, et al. Gastric and oesophageal intraepithelial lymphocytes in pediatric celiac disease. Am F Surg Pathol 1996;20:865-70.

7 De Giacomo C, Gianatti A, Negrini R, et al. Lymphocytic gastritis: a positive relationship with celiac disease. $\mathcal{F}$ Pediatr 1994;124:57-62

8 Dixon MF, Genta RM, Yardley JH, et al. Classification and grading of gastritis. The updated Sydney system. Intergrational Workshop on the Histopathology of Gastritis, Houston 1994. Am f Surg Pathol 1996;20:1161-81.

9 Armes J, Gee DC, MaCrae FA, et al. Collagenous colitis: Jejunal and colorectal pathology. F Clin Pathol 1992;45: $784-7$.

10 Moayyedi $\mathrm{P}$, O'Mahony S, Jackson $\mathrm{P}$, et al. Small intestine in lymphocytic and collagenous colitis: mucosal morphology, permeability, and secretory immunity to gliadin. F Clin Pathol 1997;50:527-9.

11 Wolber R, Owen D, DelBuono L, et al. Lymphocytic gastritis in patients with celiac sprue or spruelike intestinal disease. Gastroenterology 1990;98:310-15.

12 Feeley KM, Heneghan MA, Stevens FM, et al. Lymphocytic gastritis and coeliac disease: evidence of a positive association. 7 Clin Pathol 1998;51:207-10.

13 Dubois RN, Lazenby AJ, Yardley JH, et al. Lymphocytic enterocolitis in patients with "refractory sprue," $f A M A$ 1989;262:935-7.

14 Loft DE, Marsh MN, Crowe P. Rectal gluten challenge and diagnosis of coeliac disease. Lancet 1990;335:1293-5.

15 Hayat M, Arora DS, Dixon MF, et al. The effects of $\mathrm{H}$ pylori eradication on the natural history of lymphocytic gastritis. Gut 1998;42(suppl 1):319.

16 Lynch DAF, Sobala GM, Dixon MF, et al. Lymphocytic gastritis and associated small bowel disease: a diffuse lymphocytic gastroenteropathy? f Clin Pathol 1995;48:939-45.

17 Ferguson A, Arranz E, O'Mahony S. Clinical and pathological spectrum of coeliac disease-active, silent, latent, potential. Gut 1993;34:150-1.

18 Oberhuber G, Bodingbauer M, Mosberger I, et al. High proportion of Granzyme B-positive (activated) intraepithelial and lamina propria lymphocytes in lymphocytic gastritis. Am f Surg Pathol 1998;22:450-8. 